\title{
Homofilia, Selección e Influencia en un Estudio Longitudinal de Drogas en Población Escolar
}

\section{Homophily, Selection, and Influence in a Longitudinal Study of Drugs in School Population}

\author{
Eduardo Valenzuela y Cristián Ayala \\ Pontificia Universidad Católica de Chile
}

\begin{abstract}
Este estudio pretende establecer los niveles de homogeneidad de pares - a veces llamada homofilia en la literatura especializada - que se encuentra en el uso de tabaco, alcohol y marihuana en relaciones de amistad en la adolescencia temprana. Se utilizan datos longitudinales que identifican díadas de amistad entre alumnos de $7^{\circ}$ y $8^{\circ}$ grado provenientes de una muestra representativa de población escolarizada de la Región Metropolitana de Chile. También se ofrecen estimaciones sobre el origen de la homofilia observada, sea como resultado de procesos de selección, en los que la similitud precede a la amistad, o de influencia, en la que, por el contrario, la amistad conduce a la concordancia de pares. Los resultados muestran que la iniciación en el uso de drogas se realiza en ambientes moderadamente concordantes y que los efectos de influencia predominan sobre los de selección.
\end{abstract}

Palabras clave: drogas, efecto de pares, adolescencia

\begin{abstract}
This study seeks to establish levels of peer homogeneity — sometimes called homophily in the literature - in the use of tobacco, alcohol, and marijuana in friendship relations in early adolescence. Longitudinal data from a representative sample of school population of the Metropolitan Region of Chile was used to identify friendship dyads between students in $7^{\text {th }}$ and $8^{\text {th }}$ grades. Additionally, some estimates about the origin of the observed homophily are provided, as a result of selection processes in which similarity precedes friendship or, alternatively, as a result of influence processes where friendship leads to matching pairs. The results show that initiation into drugs takes place in environments that are moderately homogeneous and that influence effects are more important than selection effects.
\end{abstract}

Keywords: drugs, peer effect, adolescence

Según la investigación especializada, uno de los motivos más importantes de la atracción interpersonal que forma las relaciones de amistad es la similitud. La relación entre similitud y amistad ha sido documentada tanto para adultos (Berscheid \& Walster, 1985) como para adolescentes (Kandel, 1978a; Kupersmidt, DeRosier \& Patterson, 1995; Tolson \& Urberg, 1993). La similitud en los comportamientos y actitudes entre un individuo y sus pares es conocida como homofilia —equivalente a la similitud conyugal conocida como homogamia - que designa la disposición de los individuos a relacionarse con otros que son similares en varios atributos (Lazarsfeld \& Merton, 1954, citado en Prinstein \& Dodge, 2008). La interacción con individuos similares es una fuente de gratificación (Blau, 1968; Homans, 1974, ambos citados en Leenders, 1996) y, al mismo tiempo, reduce los sentimientos de hostilidad y minimiza la probabilidad de conflicto en una interacción. La semejanza alienta la aprobación del entorno social en el que se produce la amistad y, de manera especial en el caso adolescente, favorece la aceptación de los pares (Leenders, 1996). La teoría de la atracción interpersonal predice que las parejas

Eduardo Valenzuela y Cristián Ayala, Instituto de Sociología, Pontificia Universidad Católica de Chile, Santiago, Chile.

El artículo está basado en el Estudio Longitudinal de Drogas en Población Escolar (2008-2011) del Instituto de Sociología de la Pontificia Universidad Católica de Chile, el que ha contado con el apoyo de FONDECYT (Proyecto 1080054) y de la Iniciativa Científica MILENIO.

La correspondencia relativa a este artículo debe ser dirigida a Eduardo Valenzuela, Instituto de Sociología, Pontificia Universidad Católica de Chile, Avda. Vicuña Mackenna 4860, Macul, Santiago, Chile. E-mail: evalenzc@uc.cl 
homofílicas producen relaciones más estrechas, satisfactorias y estables que las que no lo son. En la investigación dinámica de la homofilia se ha mostrado que existe concordancia entre la coincidencia en determinados atributos en la adolescencia y luego en la adultez, lo que significa que las personas tienden a construir ambientes concordantes a lo largo de su vida (Kandel, Davies \& Baydar, 1990).

\section{Homofilia y Uso de Drogas Entre Adolescentes}

Los estudios en amistad adolescente encuentran niveles de homogeneidad muy altos en ciertos atributos externos, como sexo, edad y etnicidad pero, también - y de manera especial respecto de cualquier otro comportamiento-, en el uso de drogas. En uno de los estudios más comprensivos sobre esto, Kandel (1978a) encontró que el uso de drogas ofrecía la mayor tasa de homogeneidad de pares, por encima de cualquier otro comportamiento adolescente, sin contar atributos como la edad y el sexo (Haselager, Hartup, van Lieshout \& Riksen-Walraven, 1998; Kandel, 1978a; Kobus, 2003). En el caso de adolescentes, Kandel (1978a) descubre bastante similitud en rendimiento académico y en actividades de tiempo libre dentro y fuera del colegio, pero la concordancia es baja en las variables de ajuste familiar, como la cercanía con los padres (Kandel, 1978a), y en religiosidad, un atributo cuya concordancia, por el contrario, tiende a aumentar en las parejas adultas. Tolson y Urberg (1993) encuentran, asimismo, mayor homogeneidad en el uso efectivo de tabaco (iniciación y cantidad) que en cualquier otro comportamiento relacionado. Las concordancias en percepción y actitudes relacionadas con el uso de drogas entre amigos son, asimismo, muy elevadas (Akers, Jones \& Coyl, 1998), tanto o más que respecto del uso efectivo mismo. Los resultados de Kandel (1978a) son más específicos todavía y encuentran que las similitudes son mayores en el uso de drogas ilícitas, como marihuana, y tienden a ceder algo en tabaco y alcohol, lo que indica que la concordancia aumenta cuando los comportamientos no cuentan con aprobación social y las actividades ilícitas requieren de un soporte de pares, especialmente intenso, tanto en su iniciación como en su prolongación en el tiempo (Kandel, 1978a). También se ha mostrado que la concordancia en el consumo de drogas se mantiene tanto en amistades adolescentes como en cónyuges adultos (Kandel et al., 1990).

Las investigaciones sobre homofilia han mostrado recurrentemente que la amistad recíproca (aquella en que ambos se identifican mutuamente como amigos) presenta mayores niveles de similitud (Kandel, 1978b; Tolson \& Urberg, 1993). Existe evidencia de que la similitud tiende a aumentar durante la adolescencia, una vez que ciertas características internas -actitudes y valores, por ejemplo— son mejor conocidas (Urberg, Degirmencioglu \& Tolson, 1998). El proceso de selección de los amigos tiende a contar con mayor información y los ajustes se realizan con mayor exactitud a medida que se madura durante la adolescencia. Con todo, se considera que la homogeneidad es siempre mayor en conductas — o en actividades- que en actitudes y valores (Kandel, 1978a), sobre todo en la adolescencia temprana.

\section{Selección e Influencia de Pares}

La homofilia puede resultar de un proceso de selección o de influencia social (también llamado de socialización). En el primer caso, individuos con atributos similares se escogen mutuamente, de tal forma que la similitud antecede a la relación y, en algunos casos, es el motivo principal para iniciar una amistad. En el segundo caso, la homofilia es el resultado de un proceso de socialización en el que los individuos se influencian mutuamente una vez que han iniciado una determinada relación (Burk, Kerr \& Stattin, 2008; Prinstein \& Dodge, 2008). El efecto de influencia no debe confundirse con la llamada presión de pares, que es característica en las interacciones de drogas al interior de grupos de amigos (Denscombe, 2001; McIntosh, MacDonald \& McKeganey, 2006). La mayor parte de la influencia se ejerce de un modo implícito, a través de modelos y normas que indican lo que es correcto, normal o aceptable dentro de una determinada relación. En ocasiones, la sola iniciación de un amigo cercano o un grupo de amigos al 
que se quiere pertenecer puede ejercer un efecto de influencia significativo. El efecto de selección, por su parte, incluye el de de-selección, que también puede contribuir a la similitud de las relaciones de amistad. La iniciación de un determinado comportamiento —uso de drogas, por ejemplo- puede ser el motivo para romper una amistad o retirarse de un grupo que se vuelve con ello menos disímil. También se ha recalcado que estos procesos no son excluyentes y ambos pueden jugar un rol en diferentes momentos del proceso de formación, mantenimiento y disolución de la amistad (Kandel et al., 1990).

Las teorías sociológicas difieren en la importancia relativa que es necesario asignar a estos dos procesos para explicar la homofilia relacionada con comportamientos antisociales, en parte por la dificultad de separar las influencias sociales de los procesos de selección de amistad. La teoría del aprendizaje diferencial establece que el comportamiento desviado requiere aprendizaje e influencia social y se sostiene, por lo tanto, en procesos muy activos de trasmisión cultural, refuerzo social, imitación y presión de grupo. Las teorías del control social, por otra parte, consideran que el comportamiento desviado proviene de un debilitamiento de los mecanismos de integración social — desajuste familiar o fracaso escolar, por ejemplo- y que las similitudes entre amigos provienen de concordancias previas en esta clase de experiencia de desorden social (Weerman, 2011).

El efecto de influencia debe ser controlado por el efecto de selección, algo que puede hacerse con estudios longitudinales que observan cambios subsecuentes en parejas de amigos que se mantienen en el tiempo (Kandel, 1978b). Un método similar es observar iniciación en algún comportamiento (uso de drogas, por ejemplo) en parejas estables que originalmente eran disímiles en ese comportamiento (Ennett \& Bauman, 1994; Urberg, Degirmencioglu \& Pilgrim, 1997). Los estudios que parean a los individuos conforme a las percepciones tienen el inconveniente de que no pueden controlar el sesgo de sobreestimación que tienen generalmente las percepciones de similitud, por lo que es necesario basarse en auto-reportes en la declaración propia y en la de los amigos (Weerman \& Smeenk, 2005).

En el primer ciclo de investigaciones sobre homofilia - generalmente estudios transversalesla influencia de pares se consideró muy decisiva para explicar la enorme similitud — real y percibida- que se encontraba en el uso adolescente de drogas (Bauman \& Ennett, 1996). Los amigos ofrecen, efectivamente, un contexto de pares especialmente motivador para el uso de drogas, porque proporcionan un modelo de conducta y definen normas favorables hacia el consumo y son los principales proveedores de la sustancia misma. Con la proliferación de los estudios longitudinales, sin embargo, se ha logrado estimar de manera más precisa este efecto de influencia. Los estudios que son capaces de distinguir entre los efectos de influencia y selección encuentran que la influencia explica aproximadamente la mitad de la concordancia observada para díadas de mejores amigos en el uso de marihuana (Aseltine, 1995; Kandel, 1978b) o para grupos de pares en el uso de tabaco y alcohol (Bauman \& Ennett, 1996; DeVries, Candel, Engels \& Mercken, 2006; Poulin, Kiesner, Pedersen \& Dishion, 2010; Urberg et al., 1997). En una revisión sistemática de alrededor de 25 estudios que utilizan métodos avanzados para distinguir estos efectos se concluyó que casi todos los estudios encuentran a la vez efectos de socialización y selección en uso de sustancias, con una ligera inclinación a favor de los efectos de selección (Simons-Morton \& Farhat, 2010), lo que ha permitido concluir que generalmente se sobreestima la influencia de pares en la iniciación en el uso de sustancias (como tabaco, alcohol o marihuana) y que los efectos de selección son más significativos de lo que se esperaba. Se ha aportado evidencia de que los adolescentes tienden a escoger amigos más similares de los que tenían previamente y de que esas similitudes aparecen antes de iniciar una amistad (Pilgrim, Luo, Urberg \& Fang 1999; Urberg et al., 1998; Urberg, Luo, Pilgrim \& Degirmencioglu, 2003). Actualmente la evidencia respecto de efectos de socialización más bien moderados es muy robusta, aunque esto no implica que la influencia de pares sea desdeñable.

Respecto del efecto de influencia, se ha observado que: (a) opera generalmente en la dirección de estimular el consumo, antes que de inhibirlo; (b) actúa mejor a través del amigo más cercano - y en ocasiones del amigo recíproco- antes que del grupo de amigos (algo que se relaciona con el carácter ilícito del comportamiento); (c) no se intensifica con la estabilidad de 
la relación y puede aparecer tanto en relaciones recientes de amistad como en relaciones prolongadas y (d) depende de la vulnerabilidad de los adolescentes hacia procesos de influencia (en particular, la estabilidad y fortaleza de las relaciones familiares puede actuar como un factor moderador).

Existe mucho acuerdo respecto de que los comportamientos de riesgo son los más expuestos a la influencia de pares (Brechwald \& Prinstein, 2011). La influencia de los amigos respecto de comportamientos antisociales es siempre mayor que respecto de comportamientos pro-sociales, para los que se hace más caso a los padres. También se ha descubierto que se atiende más a los amigos cuando se trata de considerar las consecuencias de corto plazo de una conducta y a los padres, cuando se trata de evaluar las de largo plazo (Prinstein \& Dodge, 2008). Por otra parte, el efecto de influencia puede estar depositado en el mejor amigo (que puede impactar mejor en las actitudes y comportamiento, precisamente por la cercanía e intimidad que se tiene con él) o en el grupo de amigos (que algunas veces no incluye al mejor amigo) y que impacta, a su turno, por la fortaleza con que establece normas o pautas de conducta que cuentan con el peso de una sanción de grupo y con su capacidad para decidir la pertenencia a este. La influencia del amigo cercano se considera más poderosa que la del grupo de amigos justamente para comportamientos ilícitos, como el uso de drogas y, sobre todo, para motivar iniciación, mientras que el grupo de amigos explica mejor el uso actual o recurrente (Simons-Morton \& Farhat, 2010). El papel de la reciprocidad es más controvertido: la búsqueda de correspondencia entre amigos no recíprocos puede inducir a conformarse más intensamente con los comportamientos o actitudes del otro, pero también la calidad de la amistad recíproca tiende a reforzar la influencia de uno sobre otro, tal como ocurre en las parejas adultas (Brechwald \& Prinstein, 2011). Con todo, se ha encontrado que entre amistades no recíprocas solamente el efecto de selección explica la similitudes en el uso de tabaco, mientras que en amistades recíprocas se puede advertir con mayor claridad efectos de influencia (Mercken, Candel, Willems \& De Vries, 2007).

La evidencia respecto de una mayor vulnerabilidad de las mujeres no es concluyente (Gaughan, 2006; Urberg et al., 1997), aunque para la iniciación en tabaco se ha indicado que las mujeres son más propensas a la influencia de su mejor amiga (Simons-Morton \& Farhat, 2010). Como contrapartida, algunos estudios indican que los adolescentes hombres son más vulnerables a la influencia de pares en el uso de drogas y alcohol (Duncan, Boisjoly, Kremer, Levy \& Eccles, 2005; Erickson, Grosnoe \& Dombusch, 2000, ambos citados en Brechwald \& Prinstein, 2011). La influencia de pares está mejor documentada entre adolescentes de mayor nivel socioeconómico (NSE, Cohen \& Prinstein, 2006). La influencia que ejerce un amigo de mayor status (sea en edad o en cualquier otro atributo estatutariamente valorado) puede ser muy significativa (Blanton \& Burkley, 2008). En general, las fuentes de influencia suelen estar yuxtapuestas y ser muy interactivas (y en la adolescencia incluyen a padres, hermanos, amigos cercanos y grupo de amigos) y dependen mucho de la frecuencia y proximidad de los contactos. La capacidad de los padres de moderar la influencia de los pares es un área controvertida de la investigación (Aseltine, 1995; De Vries, Engels, Kremers, Wetzels \& Mudde, 2003), pero se sabe que mucha de la influencia parental se ejerce indirectamente a través de la selección de los amigos, mientras que la intervención parental sobre los amigos ya adquiridos tiene alguna eficacia entre las mujeres, pero casi ninguna entre los hombres (Kandel, 1996; Simons-Morton \& Farhat, 2010).

\section{Objetivos del Estudio}

El estudio que se reporta tuvo entre sus objetivos: (a) establecer niveles de homogeneidad en la iniciación en el uso de tabaco, alcohol y drogas entre parejas adolescentes de mejores amigos recíprocos y no recíprocos y comparar tales niveles con los que se observan en otros comportamientos y atributos (consultar a Aguirre, 2010 para un análisis preliminar de estos mismos datos) y (b) ofrecer evidencia acerca del dinamismo de esta concordancia, sea en la dirección de los efectos de influencia — que se pueden advertir sobre todo cuando la concordancia aumenta en parejas estables_, sea en la dirección de efectos de selección, que se determinan 
especialmente en el caso de parejas inestables que seleccionan amigos que son similares antes de iniciar la relación de amistad. Se esperaba encontrar, tal como sugiere la investigación especializada, mayores niveles de concordancia en comportamientos socialmente desaprobados, como el uso de tabaco, alcohol y marihuana - en ese orden-e indicios claros acerca de la presencia simultánea de efectos de influencia y selección de pares en el uso de estas sustancias.

\section{Método}

\section{Participantes}

La información proviene de un estudio longitudinal que ha seguido anualmente a una cohorte de estudiantes desde $7^{\circ}$ básico (2008) en adelante. El panel fue construido sobre una muestra representativa de la población escolar de la Región Metropolitana de Chile, mediante un diseño en dos etapas que contempló la selección aleatoria de establecimientos educacionales en razón del número de alumnos del nivel correspondiente (72) y la selección de cursos de $7^{\circ}$ básico (161). Asumiendo el efecto de diseño, el error muestral (varianza máxima), con un 95\% de seguridad fue 0,0146 para la muestra original.

Dentro de los cursos seleccionados fueron considerados todos los alumnos presentes en un día normal de clases, quienes contestaron un cuestionario. El resultado efectivo fueron 4.997 casos en la primera ola de mediciones. El resultado logrado en la segunda ola fue de 4.456 casos pareados, lo que arrojó un 89\% de logro. La mayor parte de los casos (3.842) fue conseguida en el mismo colegio del año anterior, pero en 614 casos la recolección se hizo en los domicilios de los alumnos. La tasa de logro fue extremadamente homogénea, según tipo de establecimiento y NSE. El estudio ha continuado con una tercera ola (2010, $1^{\circ}$ medio), pero estos datos no se incluyen en esta investigación.

\section{Instrumento}

Se aplicó un cuestionario, cuyas variables principales se detallan a continuación.

Selección de parejas de amigos. La fuente de información acerca del comportamiento de pares es doble: puede provenir del propio respondente, quien informa acerca de la conducta de sus amigos, o de auto-reportes de los propios amigos, una vez que han sido identificados por el respondente. En el primer caso, la similitud entre los amigos está sobreestimada por un efecto de proyección, que consiste en transferir actitudes y comportamientos propios a los amigos cercanos, especialmente cuando se trata de conductas indebidas que requieren de alguna normalización ("todos lo hacen"). En el segundo caso, cuando la información acerca del amigo está proporcionada por el mismo amigo, las tasas de concordancia descienden — como se ha observado en la literatura-, pero se mantienen en niveles todavía significativos (Bauman \& Fisher, 1986; Brechwald \& Prinstein, 2011).

En este estudio el cuestionario solicita la identificación (nombre, sexo y edad) de los tres mejores amigos que cada alumno tiene en el colegio. En los resultados que se presentan más adelante, sin embargo, se utiliza solamente la información acerca del mejor amigo (y se desechan las referencias del segundo y tercer mejor amigo que también han sido identificados). Debe advertirse que el mejor amigo se circunscribe a las amistades del colegio y que en algunos casos el verdadero mejor amigo puede encontrarse fuera del mismo. La identificación del mejor amigo permite parear los cuestionarios del respondente con el de su mejor amigo solamente en los casos en que esta identificación haya sido completa y fidedigna y que el amigo mencionado haya contestado el mismo cuestionario. En la primera ola se pudieron parear 3.969 cuestionarios, es decir, el 79\% del total. Las pérdidas resultan de dificultades en la identificación de amigos que no contestan el cuestionario, sea porque pertenecen a otros cursos o niveles, sea porque estaban ausentes el día de la entrevista u otra razón. En la segunda ola se lograron re-parear 2.877 cuestionarios, que corresponden al 58\% de la muestra original. 
Toda amistad implica siempre algún grado de reciprocidad, de manera que la expresión amigos recíprocos puede parecer redundante. En este estudio la reciprocidad está entendida en un sentido restringido, como aquellos que se mencionan mutuamente como mejores amigos (amigos recíprocos), lo que no significa que en las demás díadas no exista ninguna reciprocidad (el mejor amigo puede aparecer en la segunda o tercera mención o, incluso, no aparecer en una lista que se circunscribió a tres menciones y formar parte, no obstante, del grupo de amigos). Los amigos recíprocos - entendidos de esta manera - constituyen una proporción significativa, 1.862 en la primera ola y 1.180 en la segunda, los que representan un $47 \%$ y $41 \%$ del total de parejas identificadas, respectivamente.

En el análisis dinámico de la amistad se han considerado los casos de amigos que permanecen estables entre la primera y segunda ola (díadas 1 y 4 en la Tabla 3, sea porque sus atributos se miden en el primer o segundo año). Todas las demás díadas corresponden a casos de inestabilidad: la díada 2 se forma con el que dejará de ser el mejor amigo el año siguiente, mientras que la díada 3 se forma con aquel que será el mejor amigo el año entrante, ambas medidas el primer año; la díada 5 se forma con el nuevo mejor amigo y la díada 6, con aquel que dejó de ser el mejor amigo, ambas medidas en el segundo año (ver Tabla 3). En ambas olas se identifican, asimismo, los amigos recíprocos, es decir, aquellos que se nombran mutuamente como mejores amigos.

Variables independientes. El análisis de similitud contempló las variables sexo, edad, nivel educacional alcanzado por el padre y la madre (educación básica, media y superior, completa o incompleta) y religión (católica, protestante, otra o ninguna religión). La distribución por sexo está ligeramente inclinada a favor de los hombres, con una razón de 52/48.

El promedio de edad aumentó de $12,8(D E=0,70)$ a 13,8 años $(D E=0,69)$ entre ambas mediciones. El nivel educacional de los padres incluye alrededor del 18/19\% de padres/madres de baja escolaridad (educación básica o menos) y una cifra algo más inestable de padres de alta escolaridad, que oscila entre $22 \%$ en padres y $29 \%$ en madres. La proporción de respondentes católicos es muy consistente de un año a otro (63\%), mientras que los evangélicos marcan alrededor del $12 \%$.

Se incluyeron comportamientos distintos al consumo de sustancias para verificar la hipótesis acerca de la importancia que adquiere la homofilia en el uso de drogas. En algunos casos se trató de comportamientos propiamente tales, como asociatividad y hacer la cimarra (no entrar o escaparse del colegio un día de clases), mientras que en otros se trató de medidas asociadas a comportamientos (integración escolar o ajuste familiar). También se distinguió entre comportamientos positivos y negativos para anticipar la posibilidad de que existieran diferencias en la magnitud y alcance de la concordancia observada. La asociatividad positiva mide pertenencia a algún grupo que se afirma en atributos de integración social, como scouts, voluntariado, deporte, iglesias o grupos de música y arte, mientras que la asociatividad negativa mide pertenencia a alguna tribu urbana (grupos de pares que afirman explícitamente algún atributo de comportamiento desviado). Alrededor del 60\% señaló participar en alguna asociación positiva, mientras que la proporción que se reconoció como parte de alguna tribu urbana descendió de 21 a 15\% entre ambos años. Las variables de integración escolar incluyeron la disposición con que se va al colegio ("va contento al colegio"), el rendimiento escolar (mediante auto-reporte del promedio general con que termina el año escolar) y la frecuencia con que se hace la cimarra o se deja de asistir al colegio. La proporción que declara que va muy/bastante contento al colegio aumentó de 50 a 54\% entre un año y otro y la cimarra se elevó ligeramente de 17 a 20\%. Alrededor de un tercio declaró en ambos años tener un buen rendimiento escolar (promedio 6 o más). Se consideraron también dos variables de ajuste familiar: la calificación de la relación con el padre y la madre. La proporción que declaró tener una excelente relación con la madre 
(52\%) es siempre algo mayor que la que se registró con el padre (40\%) y tendió a descender levemente con la edad, de tal suerte que los valores en el segundo año se ubicaron en 48 y $36 \%$, respectivamente.

Por último, se midió consumo de sustancias a través de la prevalencia alguna vez en la vida -o iniciación- en tabaco (fumar un cigarrillo entero), alcohol (sin incluir cuando le han dado a probar o cuando ha probado frente a sus padres) y marihuana (incluso cuando ha probado solamente). La iniciación en tabaco alcanzó al 27\% de los alumnos de $7^{\circ}$ básico, incrementándose - como sucede con la progresión etaria característica en el uso de drogas- al 34,5\% en $8^{\circ}$ básico; la iniciación en alcohol progresa de 31,2 a 39,7\% y la iniciación en marihuana, de 6,7 a $11,5 \%$ en el mismo lapso.

\section{Procedimiento}

La primera ola de mediciones se realizó entre los meses de octubre y noviembre de 2008. La segunda ola fue realizada en los mismos meses del año escolar siguiente (2009), cuando la mayor parte de la muestra cursaba $8^{\circ}$ básico.

El cuestionario fue auto-aplicado. Se contó con el consentimiento expreso del director de cada establecimiento y de cada alumno en particular y el proceso fue supervisado por el Comité de Ética de FONDECYT.

\section{Análisis de Datos}

Para medir los niveles de similitud entre mejores amigos se calculó el coeficiente Kappa de Cohen. Para las variables ordinales se utilizó una matriz de ponderación lineal para capturar la magnitud de la discrepancia entre las evaluaciones (Kappa ponderado), ya que el grado de la diferencia entre dos evaluaciones crece linealmente en la medida que se alejan entre sí. Para las variables nominales no fue necesario realizar ponderaciones. Todos los datos de similitud, influencia y selección fueron analizados a nivel individual bajo la pregunta de cuán concordante son los individuos de una determinada población y cuál es el dinamismo de esa concordancia.

\section{Resultados}

Todos los coeficientes Kappa que estiman concordancia entre distintos atributos de los amigos nombrados resultaron positivos, lo que indica que siempre existe alguna propensión hacia la homofilia en la amistad (ver Tabla 1). La concordancia de género es por lejos la más elevada, lo que significa que los adolescentes tienden a tener un mejor amigo del mismo sexo, sobre todo en la adolescencia temprana. La concordancia de edad es baja porque los datos utilizados provienen de una cohorte específica del mismo nivel educacional, lo que priva de variabilidad al corte etario: el promedio de edad en 2008 para los pares de amigos fue de 12,9 años $(D E=$ $0,70)$ para un miembro y de 12,8 años $(D E=0,64)$ para el otro, mientras que en 2009 los pares de amigos tuvieron en promedio edades de $13,8(D E=0,69)$ y 13,8 años $(D E=0,66)$. La concordancia por edad sería fácilmente observable si se tomara una cohorte con mayor varianza. La educación de los padres fue un atributo de mayor concordancia, lo que indica que los amigos tienden a parecerse en términos de capital cultural (y probablemente de NSE). Los coeficientes Kappa en el estudio de Kandel (1978a) entregaron resultados de 0,13 y 0,11 para el nivel educacional alcanzado por padre y madre, respectivamente, de estudiantes norteamericanos de enseñanza media, casi tres veces menores que los que entregan los coeficientes chilenos. Estos resultados señalan que los atributos socio-demográficos $-\mathrm{y}$ en el caso chileno los atributos de clase- suelen ser los más concordantes en las díadas de amistad adolescente. 
Tabla 1

Similitudes en Características Demográficas, Comportamientos y Actitudes en Díadas de Amistad Adolescentes de $7^{\circ}$ y $8^{\circ}$ Básico (Coeficiente Kappa)

\begin{tabular}{lcc}
\hline \multicolumn{1}{c}{ Dimensión y variable } & $\begin{array}{c}\text { Año1 } \\
7^{\circ} \text { básico } \\
(2008) \\
N=3.969\end{array}$ & $\begin{array}{c}\text { Año } 2 \\
8^{\circ} \text { básico } \\
(2009) \\
N=2.876\end{array}$ \\
\hline Características socio-demográficas & & \\
$\quad$ Sexo & 0,86 & 0,85 \\
Edad & 0,09 & 0,07 \\
Educación del padre & 0,29 & 0,35 \\
Educación de la madre & 0,30 & 0,35 \\
Religión & 0,11 & 0,13 \\
Asociatividad positiva & 0,13 & 0,15 \\
Asociatividad negativa & 0,26 & 0,21 \\
Integración escolar & & \\
Va contento al colegio & 0,13 & 0,17 \\
Rendimiento escolar & 0,25 & 0,24 \\
Cimarra & 0,19 & 0,18 \\
Inasistencia escolar & 0,09 & 0,14 \\
Ajuste familiar & & \\
Relación con la madre & 0,07 & 0,06 \\
Relación con el padre & 0,06 & 0,05 \\
Uso de sustancias & & \\
Tabaco & 0,32 & 0,27 \\
Alcohol & 0,25 & 0,21 \\
Marihuana & 0,19 & 0,23 \\
\hline
\end{tabular}

Nota. Coeficientes Kappa ponderados (salvo en uso de sustancias, sexo, religión, cimarra y asociatividad). Todos los coeficientes son significativos al 0,01.

Por otra parte, la concordancia en el uso de drogas aparece bastante elevada, aunque los coeficientes son similares a los que se obtienen en otros comportamientos, como rendimiento escolar y asociatividad negativa. Los coeficientes en uso de tabaco en $7^{\circ}$ y $8^{\circ}$ grado son los más altos de la serie. La concordancia en uso de alcohol en ambos grados es importante, pero comparable con otros comportamientos, como rendimiento escolar o participación en asociaciones negativas. La concordancia en rendimiento escolar indica una cierta propensión a hacer amigos entre quienes tienen resultados académicos similares, con tasas, sin embargo, algo menores a las que se encuentran en los datos norteamericanos, que alcanzaban a 0,29 (Kandel, 1978a). Todas las variables relacionadas con ajuste familiar —en particular relaciones con los padres o tipos de familia - no son focos importantes de concordancia, tal como se ha demostrado en otras investigaciones. En el caso de drogas, los datos aparecen invertidos: se hubiera esperado que la concordancia aumentara en razón de la gravedad socialmente percibida de la sustancia involucrada: la marihuana primero y luego alcohol y tabaco. La iniciación en marihuana, sin embargo, arroja coeficientes menores en $7^{\circ}$ básico y similares al alcohol en $8^{\circ}$ básico.

Los datos también muestran que la concordancia suele ser sistemáticamente más elevada en las amistades que declaran las mujeres respecto de aquellas declaradas por los hombres (Tabla 2). En todos los atributos (con la excepción del rendimiento escolar) las mujeres tienden a concordar más con su mejor amigo/a que los hombres. Los coeficientes Kappa en tabaco, alcohol y marihuana son significativamente más altos, y en el caso de las mujeres se confirma que el uso de sustancias es el comportamiento más concordante en el abanico de conductas consideradas en este estudio (con alguna excepción en el caso de marihuana). El NSE (estimado a través de 
la educación del padre de quienes nombran a su mejor amigo) también arroja algunas disparidades en los coeficientes de concordancia. Las díadas de NSE alto suelen ser más homogéneas en varios atributos, como religión, asociatividad positiva, identificación con el colegio y rendimiento escolar, pero en comportamientos negativos esta relación se desvanece. En el uso de sustancias como tabaco, alcohol y marihuana no existe ninguna diferencia significativa en los niveles de concordancia que se obtienen en los distintos NSE.

Tabla 2

Similitudes en Características Demográficas, Comportamientos y Actitudes en Díadas de Amistad Adolescentes de $7^{\circ}$ (Año 1) y $8^{\circ}$ Básico (Año 2), Según Sexo y NSE (Coeficiente Kappa)

\begin{tabular}{|c|c|c|c|c|c|c|c|c|}
\hline \multirow{2}{*}{ Dimensión y variable } & \multicolumn{2}{|c|}{ Hombre } & \multicolumn{2}{|c|}{ Mujer } & \multicolumn{2}{|c|}{ NSE bajo } & \multicolumn{2}{|c|}{ NSE alto } \\
\hline & Año 1 & Año 2 & Año 1 & Año 2 & Año 1 & Año 2 & Año 1 & Año 2 \\
\hline \multicolumn{9}{|l|}{ Características socio-demográficas } \\
\hline Sexo & - & - & - & - & 0,87 & 0,85 & 0,84 & 0,88 \\
\hline Edad & 0,10 & 0,07 & 0,08 & 0,05 & 0,07 & $0,04 *$ & 0,11 & 0,09 \\
\hline Educación del padre & 0,25 & 0,31 & 0,33 & 0,39 & - & - & - & - \\
\hline Educación de la madre & 0,25 & 0,30 & 0,34 & 0,40 & 0,08 & 0,10 & 0,23 & 0,22 \\
\hline Religión & 0,06 & 0,12 & 0,18 & 0,14 & 0,09 & 0,10 & 0,15 & 0,18 \\
\hline Asociatividad positiva & 0,06 & 0,12 & 0,16 & 0,13 & 0,09 & 0,14 & 0,14 & 0,20 \\
\hline Asociatividad negativa & 0,19 & 0,19 & 0,31 & 0,24 & 0,22 & 0,18 & 0,29 & 0,12 \\
\hline \multicolumn{9}{|l|}{ Integración escolar } \\
\hline Va contento al colegio & 0,08 & 0,13 & 0,18 & 0,20 & 0,12 & 0,14 & 0,14 & 0,20 \\
\hline Promedio de notas auto-reportado & 0,25 & 0,22 & 0,24 & 0.26 & 0,21 & 0,20 & 0,25 & 0,27 \\
\hline Cimarra & 0,13 & 0,18 & 0,24 & 0,17 & 0,19 & 0,17 & 0,15 & 0,24 \\
\hline Inasistencia escolar & 0,07 & 0,14 & 0,11 & 0,14 & 0,10 & 0,12 & 0,08 & 0,17 \\
\hline \multicolumn{9}{|l|}{ Ajuste familiar } \\
\hline Relación con la madre & 0,04 & $0,04^{*}$ & 0,08 & 0,05 & 0,06 & $0,01^{*}$ & 0,08 & 0,11 \\
\hline Relación con el padre & $0,03^{*}$ & 0,08 & 0,09 & $0,02 *$ & 0,07 & 0,06 & 0,08 & 0,07 \\
\hline \multicolumn{9}{|l|}{ Uso de sustancias } \\
\hline Tabaco & 0,25 & 0,21 & 0,38 & 0,32 & 0,32 & 0,23 & 0,31 & 0,29 \\
\hline Alcohol & 0,17 & 0,16 & 0,33 & 0,26 & 0,23 & 0,19 & 0,22 & 0,25 \\
\hline Marihuana & 0,14 & 0,18 & 0,24 & 0,29 & 0,22 & 0,18 & 0,22 & 0,21 \\
\hline Número de casos & 2054 & 1514 & 1915 & 1362 & 1527 & 840 & 1167 & 1072 \\
\hline
\end{tabular}

Notas. Coeficientes Kappa ponderados (salvo en uso de sustancias, sexo, religión, cimarra y asociatividad). Todos los coeficientes son significativos al 0,01 salvo aquellos que se indican con *, que lo son al 0,05.

El NSE se obtuvo de la educación del padre. Bajo: educación básica incompleta a educación media incompleta; Medio: media completa a técnica profesional completa y Alto: educación universitaria.

$\mathrm{Al}$ igual que para sexo, la díada se diferencia por el NSE del respondente y no del mejor amigo nombrado.

En la tabla se omitieron los datos de personas de NSE medio, mostrando solo los dos extremos.

La homogeneidad de los amigos recíprocos en relación con los amigos no recíprocos es un resultado largamente documentado que también se registra en estos datos (Tabla 3). Los coeficientes Kappa de los amigos recíprocos son más elevados en todos los comportamientos, con la única excepción del tabaco en $8^{\circ}$ grado. En el caso de la iniciación en marihuana, por ejemplo, la concordancia entre amigos recíprocos de $7^{\circ}$ básico alcanza un coeficiente de 0,26 , mientras que la de amigos no recíprocos es solamente de 0,13 . Lo mismo ocurre en $8^{\circ}$ básico: la concordancia entre amigos recíprocos alcanza un coeficiente de 0,31 y la de amigos no recíprocos desciende a 0,18 . La homofilia entre amigos recíprocos es muy similar en uso de tabaco, alcohol y marihuana, mientras que entre amigos no recíprocos existe la gradiente ya señalada: tiende a concordarse más en tabaco que en alcohol y marihuana. 
Tabla 3

Homofilia en Uso de Drogas y Otros Atributos en Díadas de Amigos, Según Reciprocidad de la Amistad (Coeficiente Kappa)

\begin{tabular}{lcccccccc}
\hline & $N$ & Año & Tabaco & Alcohol & Marihuana & $\begin{array}{c}\text { Rendimiento } \\
\text { escolar }\end{array}$ & $\begin{array}{c}\text { Asociatividad } \\
\text { positiva }\end{array}$ & Cimarra \\
\hline $\begin{array}{l}\text { Amigos } \\
\text { recíprocos }\end{array}$ & 1862 & Año 1 & 0,38 & 0,28 & 0,26 & 0,28 & 0,17 & 0,25 \\
$\begin{array}{l}\text { Amigos no } \\
\text { recíprocos }\end{array}$ & 2107 & Año 1 & 0,26 & 0,22 & 0,13 & 0,21 & 0,09 & 0,13 \\
$\begin{array}{l}\text { Amígos } \\
\text { recíprocos }\end{array}$ & 1180 & Año 2 & 0,26 & 0,25 & 0,31 & 0,30 & 0,17 & 0,31 \\
$\begin{array}{l}\text { Amigos no } \\
\text { recíprocos }\end{array}$ & 1696 & Año 2 & 0,27 & 0,18 & 0,18 & 0,20 & 0,14 & 0,17 \\
\hline
\end{tabular}

Nota. Coeficientes Kappa no ponderados, salvo en rendimiento escolar. Todos los coeficientes son significativos al 0,01 .

Los datos dinámicos sobre homofilia en las menciones de mejores amigos entregan resultados dispares. En primer lugar, la investigación previa muestra que las parejas que permanecen estables deberían ser más parecidas que las parejas inestables, que comprende todos los casos en que se cambia de mejor amigo entre un año y otro (relación 1-2 en Tabla 4), lo que indicaría que la discordancia puede ser un motivo para cambiar de mejor amigo o, viceversa, que la concordancia promueve la mantención de la amistad. En los datos de este estudio esta regla se verifica solamente en comportamientos positivos, como rendimiento escolar y asociatividad, lo que significa que la concordancia en logro escolar o la pertenencia a ciertos grupos, como scouts o grupos deportivos, alienta la estabilidad de la amistad o, al revés, que disparidades en rendimiento o en membresía fragiliza la amistad. Sin embargo, en el caso de uso de sustancias —con la excepción de marihuana- y también en comportamientos negativos, como hacer la cimarra, se produce exactamente lo contrario: las amistades estables son menos concordantes que las inestables, lo que sugiere que la disparidad en comportamientos negativos (por ejemplo, que uno se haya iniciado en alcohol pero otro no) no es un motivo importante para abandonar la amistad.

Otro resultado característico de la investigación dinámica de la amistad es que la homofilia entre parejas estables se incrementa en el tiempo (relación 1-4), es decir, que los amigos tienden a converger entre sí en el marco de una relación de influencia mutua. Los coeficientes de concordancia, sin embargo, son casi los mismos entre amigos que permanecerán estables (medidos en el primer y segundo año), salvo en el caso de hacer la cimarra, en la que la concordancia entre amigos estables se incrementa notoriamente. También se considera que la homofilia de los nuevos amigos es mayor después de que las parejas se han formado (Año 2) que antes de formarse (Año 1; relación 5-3), lo que sugiere también un efecto de influencia. Los datos confirman esto de manera muy contundente en todos los comportamientos, sin excepción. En el caso del tabaco, por ejemplo, la concordancia entre quienes serán amigos alcanzaba a 0,18 pero, cuando se convirtieron efectivamente en amigos, esa concordancia se elevó a 0,26. En el uso de alcohol la misma relación cambia de 0,11 a 0,19 y en marihuana se aprecia un cambio mucho más drástico, de 0,05 a 0,25. Los nuevos amigos se vuelven mucho más concordantes de lo que eran antes de iniciar su amistad, lo que da indicio de un poderoso efecto de influencia que se produce, sobre todo en marihuana (y que resulta mucho más moderado en rendimiento escolar). 
Tabla 4

Homofilia en Comportamientos Seleccionados en Díadas de Amigos en Varios Estadios de Formación y Disolución de las Parejas

\begin{tabular}{|c|c|c|c|c|c|c|c|c|c|}
\hline $\mathrm{N}^{o}$ & $\begin{array}{l}\text { Estabilidad de } \\
\text { la relación }\end{array}$ & $N$ & Año & Tabaco & Alcohol & Marihuana & $\begin{array}{l}\text { Rendimiento } \\
\text { escolar }\end{array}$ & $\begin{array}{l}\text { Asociatividad } \\
\text { positiva }\end{array}$ & Cimarra \\
\hline 1 & $\begin{array}{l}\text { Estable: no } \\
\text { cambia de } \\
\text { mejor amigo }\end{array}$ & 880 & Año 1 & 0,28 & 0,21 & 0,15 & 0,26 & 0,17 & 0,13 \\
\hline 2 & $\begin{array}{l}\text { Inestable: } \\
\text { cambió de mejor } \\
\text { amigo }\end{array}$ & 1570 & Año 1 & 0,31 & 0,26 & 0,12 & 0,20 & 0,12 & 0,16 \\
\hline 3 & $\begin{array}{l}\text { Inestable: serán } \\
\text { amigos en el } \\
\text { año que viene }\end{array}$ & 1570 & Año 1 & 0,18 & 0,11 & $0,05^{*}$ & 0,19 & $0,05^{*}$ & 0,08 \\
\hline 4 & $\begin{array}{l}\text { Estable: no } \\
\text { cambió de mejor } \\
\text { amigo }\end{array}$ & 880 & Año 2 & 0,29 & 0,22 & 0,17 & 0,29 & 0,17 & 0,22 \\
\hline 5 & $\begin{array}{l}\text { Inestable: } \\
\text { nuevos amigos }\end{array}$ & 1570 & Año 2 & 0,26 & 0,19 & 0,25 & 0,22 & 0,13 & 0,15 \\
\hline 6 & $\begin{array}{l}\text { Inestable: } \\
\text { fueron amigos } \\
\text { el año anterior }\end{array}$ & 1570 & Año 2 & 0,22 & 0,20 & 0,25 & 0,15 & $0,06^{*}$ & 0,18 \\
\hline
\end{tabular}

Nota. Coeficientes Kappa no ponderados, salvo en rendimiento escolar. Todos los coeficientes son significativos, salvo aquellos que se indican con *, que lo son al 0,05 .

La investigación sobre homofilia ha encontrado, asimismo, que la homogeneidad entre ex amigos suele ser menor que entre nuevos amigos (relación 2-5), de manera que existe una predisposición a seleccionar amigos más concordantes (Kandel, 1978b; Urberg et al., 1998). Los datos de este estudio indican que los ex amigos son más y no menos concordantes que los nuevos amigos en tabaco y alcohol (aunque en marihuana ocurre lo contrario), lo que indica que estos nuevos amigos pueden ser más disímiles que los que se han perdido el año anterior. En este mismo sentido, se considera también que las parejas que se van a romper son menos similares que las que se van a formar (relación 2-3), lo que sugiere un poderoso efecto de selección, porque indica que los amigos son similares entre sí antes de iniciar una amistad. El resultado más contundente de estos datos es que esta regla no se cumple en ningún comportamiento seleccionado: en todos los casos, la concordancia con el que será el mejor amigo el año entrante es menor que la concordancia que existe con el que dejará de serlo o incluso con el que permanece siéndolo (relación 3-4), lo cual indica que las parejas que se formarán no tienen concordancias previas y que la amistad tiende a formarse desde parejas muy disímiles entre sí, lo que sugiere otra vez un efecto de influencia.

\section{Conclusiones}

Estos resultados concuerdan parcialmente con los que se encuentran en la investigación especializada. Por una parte, se observa bastante concordancia en el uso de tabaco, alcohol y marihuana - especialmente entre las mujeres-, aunque existen otros comportamientos, en particular el rendimiento escolar y la asociatividad negativa, que ofrecen mayor o igual con- 
cordancia, lo que no siempre entrega al uso de sustancias una posición especial en el análisis de similitudes. Por otra parte, los estudios han mostrado - generalmente para adolescentes de la enseñanza secundaria- que la concordancia en el uso de drogas se produce sobre todo en el caso de drogas ilícitas, mientras que en tabaco y alcohol son más moderadas (Kandel, 1978b, Urberg et al., 1998), algo que sucede exactamente al revés en los resultados de este estudio. El carácter socialmente reprobado del consumo precoz de tabaco y alcohol entrega un aire ilícito a estas sustancias — parecido al que tendrá la marihuana después_ lo que podría explicar los niveles de concordancia que se encuentran para estos comportamientos. También es posible que el uso de marihuana en la adolescencia temprana no tenga todavía la consistencia de un comportamiento socialmente desviado, con mucha necesidad de soporte y aprobación de pares, y que solo se trate de una iniciación episódica y casual.

Los datos de esta investigación muestran, asimismo, que los efectos de influencia son más consistentes que los efectos de selección. Uno de los principales argumentos a favor del efecto de selección es que, en el caso de parejas inestables, aquellos que serán amigos son muy parecidos entre sí antes de iniciar la amistad, incluso más concordantes que aquellos que dejarán de serlo, de modo que los adolescentes tienden a seleccionar amigos que son similares a ellos mismos. Los datos de este estudio muestran, sin embargo, exactamente lo contrario en todos los comportamientos que se tomaron en cuenta: aquellos que serán amigos son siempre más discordantes que los que dejarán de serlo, lo que abre las puertas para relaciones de influencia mutua. Inversamente, el principal efecto de influencia, el incremento de la concordancia entre los nuevos amigos, antes y después de serlo, queda bien documentado con estos datos, por lo menos para tabaco, alcohol y, sobre todo, para marihuana. El marco general para la iniciación en tabaco y alcohol ocurre en un ambiente de concordancia moderada y de amplia exposición hacia efectos de influencia, lo que confirma la pertinencia de los programas de prevención que se involucran decididamente en la sociabilidad de pares.

El análisis realizado en esta investigación presenta algunas limitaciones que es necesario tener en cuenta. La principal limitación es que los efectos de pares se han estimado solamente a través del mejor amigo, sin considerar otras posibilidades (los mejores amigos o el grupo de amigos, los que pueden jugar un rol igualmente determinante en el comportamiento adolescente). Estos análisis suponen también independencia en las observaciones y no se ha tenido en cuenta que algunos individuos pueden aparecer mencionados más de una vez en las díadas de amistad. Estudios más exhaustivos de relaciones de pares deberían considerar todas las relaciones actuales y potenciales dentro de las redes sociales, de manera de controlar todas las posibilidades. Por otra parte, la delimitación de los efectos de selección e influencia se ha realizado en el marco de un cuestionario con un intervalo anual, el que puede ser un período demasiado largo para estudiar modificaciones en las conductas y relaciones de amistad (por ejemplo, la concordancia actual en uso de tabaco de los nuevos amigos pudo producirse antes de que fueran amigos, lo que descartaría un efecto de influencia y permitiría presumir uno de selección). En este estudio se ha considerado solamente efectos de influencia en la iniciación, pero también podrían encontrarse estos efectos en la cesación del consumo que permitirían contrarrestar los primeros, aunque la literatura siempre ha encontrado más influencia de pares en la formación de comportamientos de riesgo que en su inhibición. Por último, los datos de concordancia se circunscriben a adolescentes que recién se inician en el uso de tabaco, alcohol y drogas ilícitas, cuando estos comportamientos no constituyen todavía un patrón de conducta establecido. Es muy probable que esta concordancia aumente significativamente con la edad y se estabilice - tal como indica la literatura especializada generalmente referida a estudiantes de enseñanza secundaria- como uno de los comportamientos que muestra mayores niveles de homogeneidad en la adolescencia. La investigación sobre homofilia y efectos de pares en comportamientos de riesgo requiere de esfuerzos ulteriores que permitan documentar niveles y patrones de concordancia en poblaciones de distintas características, precisar la dirección y magnitud de los efectos de influencia y selección y observar el papel que eventualmente pueden jugar la familia y el colegio en la moderación de la influencia de pares. 


\section{Referencias}

Aguirre, E. (2010). Amistad y homogeneidad de pares en conductas de riesgo en niños de séptimo básico de la Región Metropolitana (Tesis de habilitación profesional no publicada), Instituto de Sociología, Pontificia Universidad Católica de Chile, Santiago, Chile.

Akers, J. F., Jones, R. M. \& Coyl, D. D. (1998). Adolescent friendship pairs: Similarities in identity status development, behaviors, attitudes, and intentions. Journal of Adolescent Research, 13, 178-201. doi:10.1177/07435548981320 05

Aseltine Jr., R. H. (1995). A reconsideration of parental and peer influences on adolescent deviance. Journal of Health and Social Behavior, 36, 103-121.

Bauman, K. E. \& Ennett, S. T. (1996). On the importance of peer influence for adolescent drug use: Commonly neglected considerations. Addiction, 91, 185-198. doi:0965-2140/96/020185-14

Bauman, K. E. \& Fisher, L. A. (1986). On the measurement of the friend behavior in research on friend influence and selection: Findings from longitudinal studies of adolescent smoking and drinking. Journal of Youth and Adolescence, 15, 345-353. doi:10.1007/BF02145731

Berscheid, E. \& Walster E. H. (1985). Interpersonal attraction. Reading, MA: Addison-Wesley.

Blanton, H. \& Burkley, M. (2008). Deviance regulation theory: Applications to adolescent social influence. En M. J. Prinstein \& K. A. Dodge (Eds.), Understanding peer influence in children and adolescents (pp. 94-124). New York, NY: The Guilford Press.

Brechwald, W. A. \& Prinstein, M. J. (2011). Beyond homophily: A decade of advances in understanding peer influence processes. Journal of Research on Adolescence, 21, 166-179. doi:10.1111/j.1532-7795.2010.00721.x

Burk, W. J., Kerr, M. \& Stattin, H. (2008). The co-evolution of early adolescent friendship networks, school involvement, and delinquent behaviors. Revue Française de Sociologie, 49, 499-522.

Cohen, G. L. \& Prinstein, M. J. (2006). Peer contagion of aggression and health risk behavior among adolescent males: An experimental investigation on affects on public conduct and private attitudes. Child Development, 77, $967-983$. doi:10.1111/j.1467-8624.2006.00913.x

Denscombe, M. (2001). Peer group pressure, young people and smoking: New developments and policy implications. Drugs: Education, Prevention and Policy, 8, 7-32.

DeVries, H., Candel, M., Engels, R. \& Mercken, L. (2006). Challenges to the peer influence paradigm: Results for 12 13 year olds from six European countries from the European Smoking Prevention Framework Approach Study. Tobacco Control, 15, 83-89. doi:10.1136/tc.2003.007237

De Vries, H., Engels, R., Kremers, S., Wetzels, J. \& Mudde, A. (2003). Parents' and friends' smoking status as predictors of smoking onset: Findings from six European countries. Health Education Research, 18, 627-636. doi:10.1093/ her/cyg032

Ennett, S. T. \& Bauman, K. E. (1994). The contribution of influence and selection to adolescent peer group homogeneity: The case of adolescent cigarette smoking. Journal of Personality and Social Psychology, 67, 653-663.

Gaughan, M. (2006). The gender structure of adolescent peer influence on drinking. Journal of Health and Social Behavior, 47, 47-61. doi:10.1177/002214650604700104

Haselager, G. J. T., Hartup, W. W., van Lieshout, C. F. M. \& Riksen-Walraven, J. M. A. (1998). Similarities between friends and nonfriends in middle childhood. Child Development, 69, 1198-1208. doi:0009-3920/98/6904-0004

Kandel, D. B. (1978a). Similarity in real-life adolescent friendship pairs. Journal of Personality and Social Psychology, 36, 306-312. doi:10.1037/0022-3514.36.3.306

Kandel, D. B. (1978b). Homophily, selection, and socialization in adolescent friendships. American Journal of Sociology, 84, 427-436.

Kandel, D. B. (1996). The parental and peer contexts of adolescent deviance: An algebra of interpersonal influences. Journal of Drug Issues, 26, 289-315.

Kandel, D., Davies, M. \& Baydar, N. (1990). The creation of interpersonal contexts: Homophily in dyadic relationships in adolescence and young adulthood. En L. Robins \& M. Rutter, Straight and devious pathways from childhood to adulthood (pp. 221-241). New York, NY: Cambridge University Press.

Kobus, K. (2003). Peers and adolescent smoking. Addiction, 98(Suppl. s1), 37-55. doi:10.1046/j.1360-0443.98.s1.4.x

Kupersmidt, J. B., DeRosier, M. E. \& Patterson, C. P. (1995). Similarity as the basis for children's friendships: The roles of sociometric status, aggressive and withdrawn behavior, academic achievement and demographic characteristics. Journal of Social and Personal Relationships, 12, 439-452. doi:10.1177/0265407595123007

Leenders, R. T. A. (1996). Evolution of friendship and best friendship choices. The Journal of Mathematical Sociology, 21, 133-148. doi:10.1080/0022250X.1996.9990177

McIntosh, J., MacDonald, F. \& McKeganey, N. (2006). Why do children experiment with illegal drugs? The declining role of peer pressure with increasing age. Addiction Research \& Theory, 14, 275-287. doi:10.1080/160663505003 30465

Mercken, L., Candel, M., Willems, P. \& De Vries, H. (2007). Disentangling social selection and social influence effects on adolescent smoking: The importance of reciprocity in friendships. Addiction, 102, 1483-1492. doi:10.1111/ j.1360-0443.2007.01905.x

Pilgrim, C., Luo, Q., Urberg, K. A. \& Fang, X. (1999). Influence of peers, parents, and individual characteristics on adolescent drug use in two cultures. Merrill-Palmer Quarterly, 45, 85-107.

Poulin, F., Kiesner, J., Pedersen, S. \& Dishion, T. J. (2010). A short-term longitudinal analysis of friendship selection on early adolescent substance use. Journal of Adolescence, 34, 249-256. doi:10.1016j.2010.05.006

Prinstein, M. J. \& Dodge, K. A. (2008). Current issues in peer influence research. En M. J. Prinstein \& K. A. Dodge 
(Eds.), Understanding peer influence in children and adolescents (pp. 3-16). New York, NY: The Guilford Press. Simons-Morton, B. G. \& Farhat, T. (2010). Recent findings on peer group influences on adolescent smoking. The Journal of Primary Prevention, 31,191-208. doi:10.1007/s10935-010-0220-x

Tolson, J. M. \& Urberg, K. A. (1993). Similarity between adolescent best friends. Journal of Adolescent Research, 8, 274-288. doi:10.1037/0012-1649.31.4.540

Urberg, K. A., Degirmencioglu, S. M. \& Pilgrim, C. (1997). Close friend and group influence on adolescent cigarette smoking and alcohol use. Developmental Psychology, 33, 834-844. doi:10.1037/0012-1649.33.5.834

Urberg, K. A., Degirmencioglu, S. M. \& Tolson, J. M. (1998). Adolescent friendship selection and termination: The role of similarity. Journal of Social and Personal Relationships, 15, 703-710. doi:10.1177/0265407598155008

Urberg, K. A., Luo, Q., Pilgrim, C. \& Degirmencioglu, S. M. (2003). A two-stage model of peer influence in adolescent substance use: Individual and relationship-specific differences in susceptibility to influence. Addictive Behaviors, 28, 1243-1256. doi:10.1016/S0306-4603(02)00256-3

Weerman, F. M. (2011). Delinquent peers in context: A longitudinal network analysis of selection and influence effects. Criminology, 49, 253-286. doi:10.1111/j.1745-9125.2010.00223.x

Weerman, F. M. \& Smeenk, W. H. (2005). Peer similarity in delinquency for different types of friends: A comparison using two measurement methods. Criminology, 43, 499-524. doi:10.1111/j.0011-1348.2005.00015.x

Fecha de recepción: Mayo de 2011.

Fecha de aceptación: Septiembre de 2011. 